\title{
TITLE:
}

\section{COMPOZER-based longitudinal cross-polarization via dipolar coupling under MAS.}

\section{AUTHOR(S):}

Kamihara, Takayuki; Murakami, Miwa; Noda, Yasuto; Takeda, Kazuyuki; Takegoshi, K

\section{CITATION:}

Kamihara, Takayuki ...[et al]. COMPOZER-based longitu dinal cross-polarization via dipolar coupling under MAS.. Journal of magnetic resonance 2014, 245: 94-97

\section{ISSUE DATE:}

2014-06-24

URL:

http://hdl.handle.net/2433/189383

\section{RIGHT:}

(c) 2014 Elsevier Inc.; この論文は出版社版でありません。引用の際には 出版社版をご確認ご利用ください。; This is not the published version. Please cite only the published version. 


\title{
COMPOZER-based longitudinal cross-polarization via dipolar coupling under MAS
}

\author{
Takayuki Kamihara ${ }^{\mathrm{a}}$, Miwa Murakami ${ }^{\mathrm{b}, 1}$, Yasuto Noda ${ }^{\mathrm{a}}$, Kazuyuki Takeda ${ }^{\mathrm{a}}$, \\ K. Takegoshi ${ }^{\mathrm{a}, *}$ \\ ${ }^{a}$ Division of Chemistry, Graduate School of Science, Kyoto University, \\ Kitashirakawa-Oiwakecho, Sakyo-ku, Kyoto, Japan 606-8502 \\ ${ }^{b}$ National Institute for Materials Science, 3-13 Sakura, Tsukuba, Ibaraki, Japan 305-0003
}

\begin{abstract}
We propose a cross polarization $(\mathrm{CP})$ sequence effective under magic-angle spinning (MAS) which is tolerant to RF field inhomogeneity and Hartmann-Hahn mismatch. Its key feature is that spin locking is not used, as CP occurs among the longitudinal $(Z)$ magnetizations modulated by the combination of two pulses with the opposite phases. We show that, by changing the phases of the pulse pairs synchronized with MAS, the flip-flop term of the dipolar interaction is restored under MAS.

Keywords: cross polarization, flip-flop exchange, magic-angle spinning

Cross polarization $(\mathrm{CP})$ is a technique often used to overcome the low sensitivity of dilute spins, e.g. ${ }^{13} \mathrm{C}$, by magnetization transfer from abundant spins, e.g. ${ }^{1} \mathrm{H}$. In general, CP requires both spin locking and Hartmann-Hahn matching $[\mathbb{\Pi},[2]$. The basic $\mathrm{CP}$ sequence employs continuous-wave $(\mathrm{CW})$ radio-
\end{abstract}

\footnotetext{
* Corresponding author: Division of Chemistry, Graduate School of Science, Kyoto University, Kitashirakawa-Oiwakecho, Sakyo-ku, Kyoto, Japan 606-8502, Tel.: +81-75-753-4015

Email address: takeyan@kuchem.kyoto-u.ac.jp (K. Takegoshi)

${ }^{1}$ Current address: Office of Society-Academia Collaboration for Innovation (SACI), Kyoto University, Sakyo-ku, Kyoto, Japan 606-8501
} 
field (RF) irradiation for spin locking. Levitt et al. suggested a modification to the spin-locking pulse, known as mismatch-optimized $I$-S transfer (MOIST) [3], to alleviate the effect of RF field inhomogeneities by repeated phase inversions. Later, the phase inversion scheme was combined with magic angle spinning (MAS), leading to a sequence referred to as simultaneous phase-inversion CP (SPICP) [4, 5]. Since then, various CPMAS methods with amplitude-, phaseand/or frequency-modulation have been proposed. They include variable-amplitude cross polarization (VACP) [6], amplitude-modulated cross polarization (AMCP) [7], ramped-amplitude cross polarization (RAMP-CP) [8] , frequency sweep cross polarization (FSCP) [9], nuclear integrated cross polarization (NICP) [III], and simultaneous adiabatic spin-locking cross polarization (SADIS CP)[П]].

Recently, we proposed a CP sequence which does not use the spin-locking pulse but instead employs a series of phase-inverted $2 \pi$ pulses. This method, called composite zero-degree pulse CP (COMPOZER[एँ2]), induces transfer of longitudinal $(Z)$ magnetizations. Even though this unique $\mathrm{CP}$ technique was shown to be effective for static samples with tolerance against RF field inhomogeneity and Hartmann-Hahn mismatch, its CP efficiency almost diminishes under MAS where the heteronuclear dipolar interaction gains time dependence.

In this paper, we propose a $\mathrm{CP}$ scheme under MAS which retains the merits of COMPOZER, such as tolerance for RF field inhomogeneity and robustness against Hartmann-Hahn mismatch. This method employs phase modulation in such a way that the relevant parts of the dipolar Hamiltonian gains additional time dependence by spin rotation around the $Z$ axis in the interaction frame. 
For this reason, we call the new $\mathrm{CP}$ sequence as COMPOZER with $Z$ rotation $(\mathrm{CPZ})$.

We consider a heteronuclear two spin system $(I$ and $S)$, whose dipolar interaction in the conventional rotating frame is given by the Hamiltonian,

$$
\mathcal{H}_{d}=d I_{z} S_{z}
$$

$d$ denotes the geometrical part of the heteronuclear dipolar interaction given by

$$
d=-\frac{\mu_{0}}{4 \pi} \frac{\gamma_{\mathrm{I}} \gamma_{\mathrm{S}} \hbar}{r_{\mathrm{IS}}^{3}}\left(3 \cos ^{2} \theta-1\right),
$$

where $\gamma_{\mathrm{X}}$ represents the gyromagnetic ratio for the $X$ spin, and $r_{\mathrm{IS}}$ and $\theta$ are the internuclear $I-S$ distance and the angle between external magnetic field and the $I-S$ vector, respectively. $\mu_{0}$ is the vacuum permeability, thus the constant $\mu_{0} / 4 \pi$ represents Eq. (『) to be in SI units.

The basic sequence of COMPOZER is written as $-(2 \pi)_{X}-(2 \pi)_{Y}-$, where $(2 \pi)_{\phi}$ denotes two $2 \pi$ pulses with a phase $\phi$ applied to both $I$ and $S$ spins simultaneously. The zeroth order average Hamiltonian over two $2 \pi$ pulse periods is given by[[12],

$$
\overline{\widetilde{\mathcal{H}}_{d}}=\frac{d}{8}\left\{4 I_{z} S_{z}+\left(I^{+} S^{-}+I^{-} S^{+}\right)\right\}
$$

The flip-flop term, $I^{+} S^{-}+I^{-} S^{+}$, indicates that CP occurs between the $Z$ magnetizations. Under MAS, however, $d$ becomes time dependent;

$$
d \rightarrow d(t)=D_{1} \cos \left(\omega_{r} t+\psi\right)+D_{2} \cos \left(2 \omega_{r}+2 \psi\right),
$$


with

$$
D_{1}=\sqrt{2} \frac{\mu_{0}}{4 \pi} \frac{\gamma_{\mathrm{I}} \gamma_{\mathrm{S}} \hbar}{r_{\mathrm{IS}}^{3}} \sin 2 \beta
$$

and

$$
D_{2}=-\frac{\mu_{0}}{4 \pi} \frac{\gamma_{\mathrm{I}} \gamma_{\mathrm{S}} \hbar}{r_{\mathrm{IS}}^{3}} \sin ^{2} \beta
$$

where $\omega_{r}$ represents the MAS rate, and $\beta$ and $\psi$ are a polar angle and an azimuthal angle that orient the $I-S$ vector $\mathbf{r}_{I S}$ with respect to the MAS rotation axis. Eq. (四) that $d(t)$ is averaged out under MAS, leading to inefficient CP.

The basic idea of modification is to add rotation around the $Z$ axis to the spin part of the Hamiltonian,

$$
\begin{aligned}
\overline{\mathcal{H}_{d}} & =d(t) \times e^{i \omega\left(I_{z}-S_{z}\right) t}\left(I^{+} S^{-}+I^{-} S^{+}\right) e^{-i \omega\left(I_{z}-S_{z}\right) t} \\
& =d(t) \times\left(e^{-i 2 \omega t} I^{+} S^{-}+e^{i 2 \omega t} I^{-} S^{+}\right) .
\end{aligned}
$$

It becomes possible to interfere the MAS averaging by matching the $Z$ rotation rate $\omega$ to $\omega_{r} / 2$ or $\omega_{r}$. In this work, we rotate the phase of the $R F$ pulses to realize the $Z$ rotation. The basic sequence of $\mathrm{CPZ}$ consists of pairs of phase alternating two pulses $\theta_{\phi}-\theta_{\bar{\phi}}$ (Fig. 1(a)), where $\theta_{\phi}$ denotes a pulse with the flip angle of $\theta$ and a phase of $\phi$. Similar to COMPOZER, the $\theta_{\phi}-\theta_{\bar{\phi}}$ pulse pair is applied to both $I$ and $S$ spins simultaneously.

The CPZ sequence consists of $N$ pairs of the phase-alternated pulse blocks for every two rotor periods. The RF irradiation phase of the $n$-th pair, $\Phi_{n}=$ 
(a)
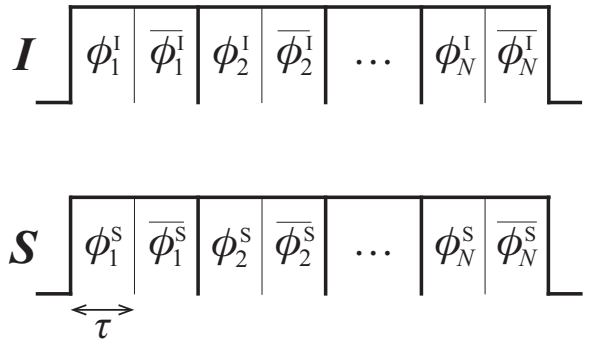

(b)

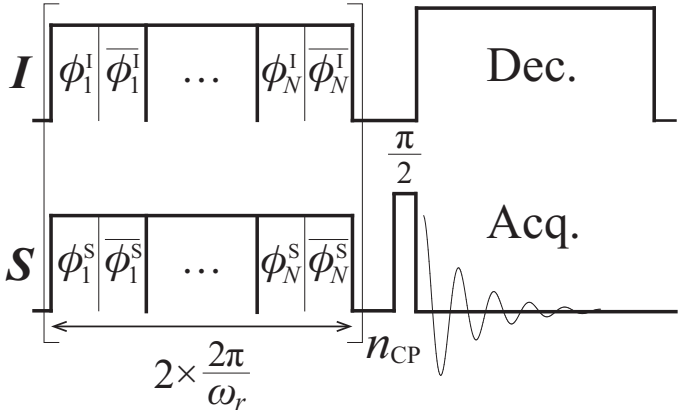

Fig. 1: Pulse sequences for CPZ: (a) pairs of two pulses with phase alternation and (b) the CPZ sequence followed by a single pulse sequence with $I$-spin decoupling. The inverted phase $\overline{\phi_{n}^{\mathrm{X}}}=\phi_{n}^{\mathrm{X}}+\pi$ denotes the RF phase for the $X(X=I$ or $S)$ spin. 
$\left(\phi_{n}^{\mathrm{I}}, \phi_{n}^{\mathrm{S}}\right)(n=1, \cdots, N)$, is set to be

$$
\begin{aligned}
& \phi_{n}^{\mathrm{I}}=-2 \pi \times \frac{n}{N}+(n-1) \pi, \\
& \phi_{n}^{\mathrm{S}}=2 \pi \times \frac{n}{N}
\end{aligned}
$$

where $\phi_{n}^{\mathrm{X}}$ denotes the RF phase for the $X(X=I$ or $S)$ spin. Note that the opposite sign for $\phi_{n}^{\mathrm{I}}$ and $\phi_{n}^{\mathrm{S}}$ reflects the opposite direction of the $Z$ rotation for $I$ and $S$ in Eq. (ם). The propagator for the RF irradiation with the phase $\Phi_{n}$, $U_{\mathrm{RF}}^{\Phi_{n}}(t)$, is written as

$$
\begin{aligned}
U_{\mathrm{RF}}^{\Phi_{n}}(t) & =e^{-i \omega_{1 \mathrm{I}}\left(I_{x} \cos \phi_{n}^{\mathrm{I}}-I_{y} \sin \phi_{n}^{\mathrm{I}}\right) t} e^{-i \omega_{1 \mathrm{~S}}\left(S_{x} \cos \phi_{n}^{\mathrm{S}}-S_{y} \sin \phi_{n}^{\mathrm{S}}\right) t} \\
& =e^{-i \phi_{n}^{\mathrm{I}} I_{z}} e^{-i \omega_{1 \mathrm{I}} I_{x} t} e^{i \phi_{n}^{\mathrm{I}} I_{z}} \cdot e^{-i \phi_{n}^{\mathrm{S}} S_{z}} e^{-i \omega_{1 \mathrm{~S}} S_{x} t} e^{i \phi_{n}^{\mathrm{S}} S_{z}}
\end{aligned}
$$

where $\omega_{1 \mathrm{I}}$ and $\omega_{1 \mathrm{~S}}$ are $\mathrm{RF}$ nutation frequencies of $I$ and $S$ spin, respectively. 
The dipolar Hamiltonian in the interaction frame of RF irradiation is given by

$$
\begin{aligned}
& \widetilde{\mathcal{H}}_{d}^{\Phi_{n}}(t) \\
= & \left\{U_{\mathrm{RF}}^{(n)}(t)\right\}^{-1} \mathcal{H}_{d} U_{\mathrm{RF}}^{(n)}(t) \\
= & \frac{d(t)}{2} \\
& \times\left\{I_{z} S_{z}(\cos \Delta t+\cos \Sigma t)\right. \\
& +\left(\sin \phi_{n}^{\mathrm{I}} \sin \phi_{n}^{\mathrm{S}} I_{x} S_{x}-\sin \phi_{n}^{\mathrm{I}} \cos \phi_{n}^{\mathrm{S}} I_{x} S_{y}\right. \\
& \left.\quad-\cos \phi_{n}^{\mathrm{I}} \sin \phi_{n}^{\mathrm{S}} I_{y} S_{x}+\cos \phi_{n}^{\mathrm{I}} \cos \phi_{n}^{\mathrm{S}} I_{y} S_{y}\right)(\cos \Delta t-\cos \Sigma t) \\
& +\left(-\sin \phi_{n}^{\mathrm{I}} I_{x} S_{z}+\cos \phi_{n}^{\mathrm{I}} I_{y} S_{z}\right)(\sin \Delta t+\sin \Sigma t) \\
& \left.\quad\left(-\sin \phi_{n}^{\mathrm{S}} I_{z} S_{x}+\cos \phi_{n}^{\mathrm{S}} I_{z} S_{y}\right)(\sin \Delta t-\sin \Sigma t)\right\},
\end{aligned}
$$

where $\Sigma$ and $\Delta$ are the sum $\Sigma=\omega_{1 \mathrm{I}}+\omega_{1 \mathrm{~S}}$ and the difference $\Delta=\omega_{1 \mathrm{I}}-\omega_{1 \mathrm{~S}}$ of the RF amplitudes, respectively. For the phase-inverted pair of pulses, the Hamiltonian, $\widetilde{\mathcal{H}}_{d}^{\overline{\Phi_{n}}}(t)$, can be obtained in the same manner, and we assume that the zeroth order average Hamiltonian for $\theta_{\Phi_{n}}-\theta_{\overline{\Phi_{n}}}$ is given simply by

$$
\overline{\widetilde{\mathcal{H}}_{d}^{(n)}}=\frac{1}{2 \tau}\left\{\int_{2(n-1) \tau}^{(2 n-1) \tau} \widetilde{\mathcal{H}}_{d}^{\Phi_{n}}(t) d t+\int_{(2 n-1) \tau}^{2 n \tau} \widetilde{\mathcal{H}}_{d}^{\Phi_{n}}(t) d t\right\},
$$

where $\tau$ is the pulse width. Note that the pulse width $\tau$ is given by

$$
\tau=\frac{\tau_{r}}{N}
$$

with $\tau_{r}$ being the rotor period $\left(\tau_{r}=2 \pi / \omega_{r}\right)$. The flip angle of the pulse $\theta_{\mathrm{X}}(X$ 
$=I$ or $S)$ is therefore written as

$$
\theta_{\mathrm{X}}=\omega_{1 \mathrm{X}} \frac{\tau_{r}}{N} .
$$

We found that for the case of a rotor cycle divided into eight (Fig. 1(b)]), i.e., $N=8$, the "sin $\Delta t$ " and "sin $\Sigma t$ " terms in Eq. (ㅁ) vanish after averaging over the 16 pulse periods (two rotor cycles). The total average Hamiltonian for the 8 pulse pairs is given by

$$
\begin{aligned}
\overline{\widetilde{\mathcal{H}}_{d}} & =\frac{1}{8} \sum_{n=1}^{8} \overline{\widetilde{\mathcal{H}}_{d}^{(n)}} \\
& =f(\Delta, \Sigma) I_{z} S_{z}+g_{1}(\Delta, \Sigma) I^{+} S^{-}+g_{2}(\Delta, \Sigma) I^{-} S^{+},
\end{aligned}
$$

where $f(\Delta, \Sigma), g_{1}(\Delta, \Sigma)$ and $g_{2}(\Delta, \Sigma)$ are functions of $\Delta$ and $\Sigma$. The exact formulae for $f$ and $g$ as well as detailed calculation of the above derivations will be reported elsewhere.

At $\Delta=0$, Eq. (ㅍ.5) becomes

$$
\overline{\widetilde{\mathcal{H}}_{d}}=\frac{\sqrt{2} D_{1}}{8 \pi} \cdot e^{i \frac{3}{4} \pi S_{z}}\left(I^{+} S^{-}+I^{-} S^{+}\right) e^{-i \frac{3}{4} \pi S_{z}},
$$

with ignoring the $\Sigma$ terms, whose contribution will be negligible when $\Sigma \gg \omega_{r}$. Eq. ([6]) shows that with the CPZ irradiation the flip-flop term is restored under MAS, which enables $\mathrm{CP}$ between the $Z$ magnetizations. The $\Delta=0$ condition means the equal pulse flip angle for $I$ and $S, \theta_{\mathrm{I}}=\theta_{\mathrm{S}}$. It is true that the recoupling mechanism is not directly related to the original Hartmann-Hahn 
matching, but we shall call the condition as the Hartmann-Hahn condition in the following for ease of legibility.

We demonstrated the CPZ experiment for the ${ }^{1} \mathrm{H}^{-13} \mathrm{C}$ system using adamantane. All NMR spectra were obtained at room temperature in a magnetic field of 9.4 T with Larmor frequencies of $400.2 \mathrm{MHz}$ and $100.6 \mathrm{MHz}$ for the ${ }^{1} \mathrm{H}$ and ${ }^{13} \mathrm{C}$ nuclei, respectively, on an OPENCORE spectrometer[13]. A Chemagnetics T3 MAS probe for a $3.2 \mathrm{~mm}$ rotor was used. The MAS frequency $\omega_{r} / 2 \pi$ was set to $20.00 \mathrm{kHz}$. The RF amplitudes for both ${ }^{1} \mathrm{H}$ and ${ }^{13} \mathrm{C} 90^{\circ}$ pulses were about $100 \mathrm{kHz}$ and that for the ${ }^{13} \mathrm{C} \mathrm{CPZ}$ pulses was $50 \mathrm{kHz}$. The ${ }^{1} \mathrm{H} \mathrm{RF}$ amplitude for $\mathrm{CPZ}$ was used as an experimental parameter as shown below. The pulse width and phase-modulation angle for TPPM decoupling with the RF amplitude of ca. $70 \mathrm{kHz}$ were $11 \mu \mathrm{s}$ and $\pm 17.5^{\circ}$, respectively. The recycle delay used was 4 $\mathrm{s}$ in all experiments. The carrier frequencies were set at on-resonance for ${ }^{1} \mathrm{H}$ in solid adamantane (1.8 ppm) and for ${ }^{13} \mathrm{C}$ of the methylene carbons $(38.6 \mathrm{ppm})$ from TMS, respectively.

The pulse width $\tau$ for the one of the pulses in the basic pair of CPZ was $6.25 \mu \mathrm{s}$, which corresponds to one-eighth of the MAS rotation cycle $(50.0 \mu \mathrm{s})$ and the pulse-flip angle $\theta$ of $112.5^{\circ}$. The phase alternation cycle for one $\mathrm{CPZ}$ block (8 pairs) was $\left\{45^{\circ}, 225^{\circ}, 90^{\circ}, 270^{\circ}, 135^{\circ}, 315^{\circ}, 180^{\circ}, 360^{\circ}, 225^{\circ}, 45^{\circ}, 270^{\circ}\right.$, $\left.90^{\circ}, 315^{\circ}, 135^{\circ}, 360^{\circ}, 180^{\circ}\right\}$ for the ${ }^{13} \mathrm{C}$ channel and $\left\{-45^{\circ},-225^{\circ},-270^{\circ}\right.$, $-90^{\circ},-135^{\circ},-315^{\circ},-360^{\circ},-180^{\circ},-225^{\circ},-45^{\circ},-90^{\circ},-270^{\circ},-315^{\circ},-135^{\circ}$, $\left.-180^{\circ},-360^{\circ}\right\}$ for the ${ }^{1} \mathrm{H}$ channel, respectively. The $\mathrm{CPZ}$ sequence may be written in the Levitt's symmetry-based notation[14] as $C 8_{2}^{5}$ and $C 8_{2}^{1}$ for the $I$ 
and $S$ spins, respectively. Note that the cyclicity in this sequence is achieved for the pair of alternating phase (Eq. (एव))

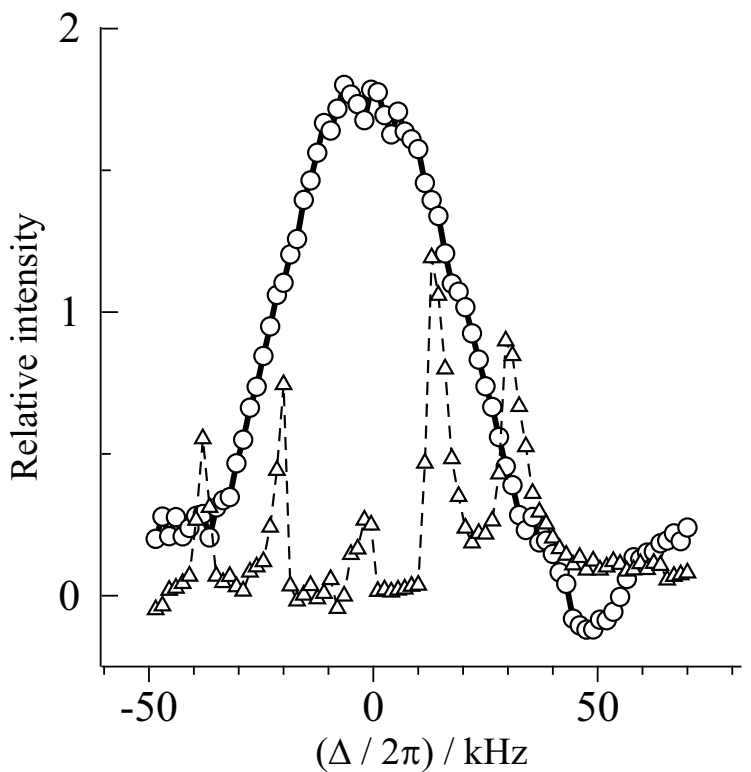

Fig. 2: CP profiles against the Hartmann-Hahn mismatch under MAS $\left(\omega_{r} / 2 \pi=20 \mathrm{kHz}\right)$. Circles and triangles indicate the intensity of the $\mathrm{CH}_{2}$ carbons of adamantane obtained from $\mathrm{CPZ}$ and conventional $\mathrm{CP}$, respectively. The $\mathrm{RF}$ amplitude for the ${ }^{13} \mathrm{C}$ channel was ca. 50 $\mathrm{kHz}$ in both experiments. The signal intensities were normalized by the thermal equilibrium intensity. The number of CPZ blocks, $n_{\mathrm{CP}}$, was set to be 160 , corresponding to the contact time of $16 \mathrm{~ms}$. The contact time for conventional $\mathrm{CP}$ was $8 \mathrm{~ms}$. The solid and the dotted lines through the data points are for eye-guidance.

Fig. $\square$ compares the performance of $\mathrm{CPZ}$ and conventional $\mathrm{CP}$ against the Hartmann-Hahn $(\mathrm{H}-\mathrm{H})$ mismatch $(\Delta / 2 \pi \mathrm{kHz})$ under MAS $\left(\omega_{r} / 2 \pi=20 \mathrm{kHz}\right)$ for the ${ }^{13} \mathrm{CH}_{2}$ peak in adamantane. In the $\mathrm{CPZ}$ experiment, we suppressed the ${ }^{13} \mathrm{C}$ magnetization recovered in the recycle delay by a saturation pulse train before the $\mathrm{CP}$ sequence. This was to compare the magnetization build-up curves for CPZ and conventional CP (Fig. [3). In its practical use, the presaturation is not required.

Fig. [ shows that the maximum $\mathrm{CP}$ efficiency can be attained at the $\mathrm{H}-\mathrm{H}$ 
matching condition $(\Delta=0)$ for CPZ. For conventional CP, the H-H matching is achieved at the so-called sideband condition, $\Delta= \pm n \omega_{r} / 2 \pi(n=1,2)$. It is notable that the signal intensities for conventional CP were relatively poor, which is ascribed to RF field inhomogeneity over the sample volume. Since CPZ employs the phase-alternated pair as its basic pulse, the effects of RF field inhomogeneity is less critical. Clearly, CPZ bears higher tolerance for $\mathrm{H}-\mathrm{H}$ mismatch and for RF field inhomogeneity compared with conventional CP.

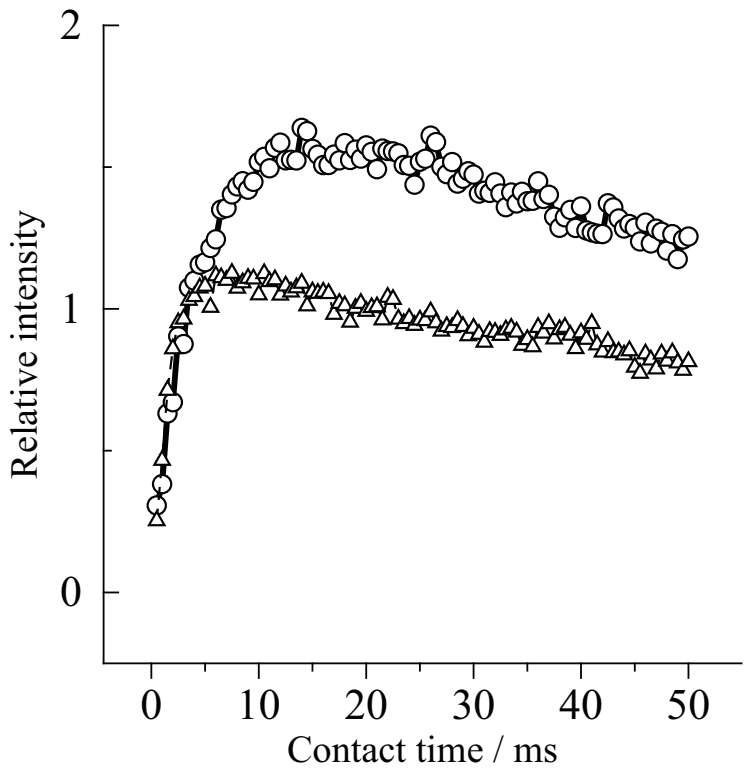

Fig. 3: CP build-up curves under MAS $\left(\omega_{r} / 2 \pi=20 \mathrm{kHz}\right)$. Circles and triangles indicate the intensity of the $\mathrm{CH}_{2}$ carbons of adamantane obtained under $\mathrm{CPZ}$ and conventional $\mathrm{CP}$, respectively. The $\mathrm{RF}$ amplitude for the ${ }^{13} \mathrm{C}$ channel was ca. $50 \mathrm{kHz}$ in both experiments. The Hartmann-Hahn mismatches $(\Delta / 2 \pi)$ were 0 for $\mathrm{CPZ}$ and $20 \mathrm{kHz}$ for conventional CP, respectively. The signal intensities were normalized by the thermal equilibrium intensity. The solid and the dotted lines through the data points are for eye-guidance.

In Fig. 3, we compare the $\mathrm{CP}$ build-up curves for the ${ }^{13} \mathrm{CH}_{2}$ peak in adamantane for $\mathrm{CPZ}$ and conventional $\mathrm{CP}$. The magnetization-transfer rate under conventional CP seems slightly faster than that under $\mathrm{CPZ}$, which can be attributed 
to difference of the sizes of the average dipolar Hamiltonian. For CPZ, the size is given by $\widetilde{\mathcal{H}}_{d}$ in Eq. (ㅁ) as $\sqrt{2} D_{1} / 8 \pi$. For conventional $\mathrm{CP}$ at the sideband condition $\Delta=\omega_{r} / 2 \pi, \widetilde{\mathcal{H}}_{d}$ is given in the tilted frame by

$$
\overline{\widetilde{\mathcal{H}}_{d}^{T}}=\frac{D_{1}}{8}\left(I^{+} S^{-}+I^{-} S^{+}\right)
$$

Here the size is $D_{1} / 8$ for conventional CP. In other words, the size of the CPZ Hamiltonian is smaller than that of conventional CP by $\sqrt{2} / \pi$. In fact, Fig. 3] shows that the time to achieve their local maxima for both sequence are $14 \mathrm{~ms}$ for $\mathrm{CPZ}$ and $6 \mathrm{~ms}$ for conventional CP, whose ratio is about $\sqrt{2} / \pi$. The dependence of the $\mathrm{CP}$ build-up curves on the pulse flip angle $\theta$, i.e., the $\mathrm{RF}$ amplitude and the Hartmann-Hahn mismatch $\Delta$ will be examined the forthcoming full paper.

To summarize, we described a CP scheme under MAS, CPZ, based on COMPOZER. The original COMPOZER works efficiently under the static condition, while its efficacy decreases under the MAS condition. Since the decrement is brought about by the geometrical rotation, the additional rotation to the spin part of the COMPOZER Hamiltonian would recover the CP efficacy. In this work, we showed that the rotation of the phase of RF irradiation synchronous to the MAS rotation successfully realizes the required $Z$ rotation and the CPZ sequence thus developed retains the merit of COMPOZER under MAS.

Lastly, we would like to point out three aspects of CPZ. One is a potential limitation derived from magnetization decay during the CP period; for conventional $\mathrm{CP}$, the magnetization decays with a time constant $T_{1 \rho}$, while for $\mathrm{CPZ}$, 
it decays with what may be called $T_{2 \rho}$. Another is a limitation in RF amplitude. Although the recoupling condition of $\mathrm{CPZ}\left(\theta_{\mathrm{I}}=\theta_{\mathrm{S}}\right)$ is independent of RF amplitude, the $\Sigma$ terms, which were neglected in Eq. ([ए6), becomes appreciable when $\Sigma \leq \omega_{r}$. As the signs of the $\Sigma$ term and the $\Delta$ term are opposite, the size of the recoupled CPZ Hamiltonian is reduced, leading to inefficient CP. Hence, $\Sigma>\omega_{r}$ is a mandatory condition. The third point is that CPZ has a potential merit of the tolerance to fluctuation of the anisotropic interaction along the $Z$ direction brought about by MAS, which makes the spin locking difficult[15, [6]. Since magnetization transfer occurs among the $Z$ magnetizations under MAS, CPZ is not significantly affected by the $Z$ fluctuations. CPZ would therefore be applicable for nuclei with large anisotropies, i.e., chemical shift anisotropy and quadrupolar interaction. Application of CPZ to a system with large anisotropies, such as ${ }^{19} \mathrm{~F}_{-}{ }^{13} \mathrm{C}$, and a new sequence, which employs the resonance offset as the source of the $Z$ rotation, will be given in the forthcoming full paper.

\section{References}

[1] A. Pines, M.G. Gibby, J.S. Waugh, Proton-enhanced NMR of dilute spins in solids, J. Chem. Phys. 59 (1973) 569-590. DOI: http://dx.doi.org/10.1063/1.1680061.

[2] J. Schaefer, E.O. Stejskal, Carbon-13 nuclear magnetic resonance of polymers spinning at the magic angle, J. Am. Chem. Soc. 98 (1976) 1031-1032. DOI: http://dx.doi.org/10.1021/ja00420a036. 
[3] M.H. Levitt, D. Suter, R.R. Ernst, Spin dynamics and thermodynamics in solid-state NMR cross polarization, J. Chem. Phys. 84 (1986) 4243-4255. DOI: http://dx.doi.org/10.1063/1.450046.

[4] T.M. Barbara, E.H. Williams, Modulated sequences for cross polarization during high-speed MAS, J. Magn. Reson. 99 (1992) 439-442. DOI: http://dx.doi.org/10.1016/0022-2364(92)90200-Q.

[5] X. Wu, K.W. Zilm, Cross polarization with high-speed magicangle spinning, J. Magn. Reson. A 104 (1993) 154-165. DOI: http://dx.doi.org/10.1006/jmra.1993.1203.

[6] O.B. Peersen, X. Wu, I. Kustanovich, S.O. Smith, Variable-amplitude cross-polarization MAS NMR, J. Magn. Reson. A 104 (1993) 334-339. DOI: http://dx.doi.org/10.1006/jmra.1993.1231.

[7] S. Hediger, B.H. Meier, R.R. Ernst, Cross polarization under fast magic angle sample spinning using amplitude-modulated spin-lock sequences, Chem. Phys. Lett. 213 (1993) 627-635. DOI: http://dx.doi.org/10.1016/00092614(93)89172-E.

[8] G. Metz, X. Wu, S.O. Smith, Ramped-amplitude cross polarization in magic-angle-spinning NMR, J. Magn. Reson. A 110 (1994) 219-227. DOI: http://dx.doi.org/10.1006/jmra.1994.1208.

[9] A.C. Kolbert, A. Bielecki, Broadband Hartmann-Hahn matching in magicangle spinning NMR via an adiabatic frequency sweep, J. Magn. Reson. A 116 (1995) 29-35. DOI: http://dx.doi.org/10.1006/jmra.1995.1186. 
[10] W.K. Peng, K. Takeda, M. Kitagawa, A new technique for cross polarization in solid-state NMR compatible with high spinning frequencies and high magnetic fields, Chem. Phys. Lett. 417 (2006) 58-62. DOI: http://dx.doi.org/10.1016/j.cplett.2005.10.012.

[11] W.K. Peng, A. Samoson, M. Kitagawa, Simultaneous adiabatic spin-locking cross polarization in solid-state NMR of paramagnetic complexes, Chem. Phys. Lett. 460 (2008) 531-535. DOI: http://dx.doi.org/10.1016/j.cplett.2008.06.027.

[12] M. Fukuchi, A. Ramamoorthy, K. Takegoshi, Efficient cross-polarization using a composite $0^{\circ}$ pulse for NMR studies on static solids, J. Magn. Reson. 196 (2009) 105-109. DOI: http://dx.doi.org/10.1016/j.jmr.2008.10.013.

[13] K. Takeda, OPENCORE NMR: Open-source core modules for implementing an integrated FPGA-based NMR spectrometer, J. Magn. Reson. 192 (2009) 218-229. DOI: http://dx.doi.org/10.1016/j.jmr.2008.02.019.

[14] M.H. Levitt, Symmetry-Based Pulse Sequences in Magic-Angle Spinning Solid-State NMR, eMagRes (2007).

[15] A.J. Vega, MAS NMR spin locking of half-integer quadrupolar nuclei, J. Magn. Reson. 96 (1992) 50-68. DOI: http://dx.doi.org/10.1016/00222364(92)90287-H.

[16] A.J. Vega, CP/MAS of quadrupolar $S=3 / 2$ nuclei, Solid State Nucl. Magn. Reson. 1 (1992) 17-32. DOI: http://dx.doi.org/10.1016/09262040(92)90006-U. 